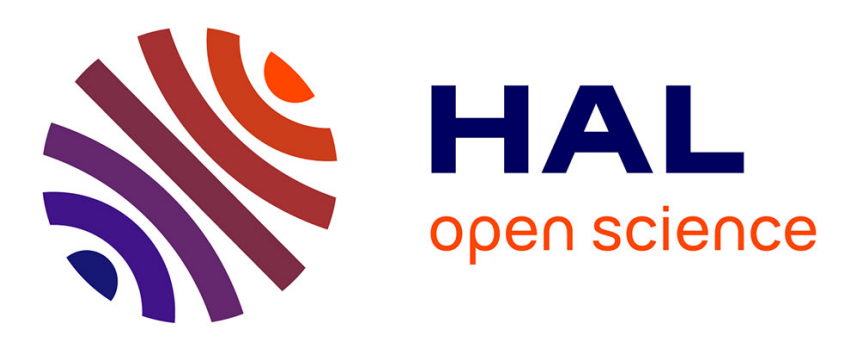

\title{
DX centers in AlAs and GaAs-AlAs selectively doped superlattices
}

\author{
S. Ababou, J. Marchand, L. Mayet, G. Guillot, F. Mollot
}

\section{To cite this version:}

S. Ababou, J. Marchand, L. Mayet, G. Guillot, F. Mollot. DX centers in AlAs and GaAs-AlAs selectively doped superlattices. Journal de Physique III, 1991, 1 (7), pp.1301-1309. 10.1051/jp3:1991190 . jpa-00248658

\section{HAL Id: jpa-00248658 https://hal.science/jpa-00248658}

Submitted on 1 Jan 1991

HAL is a multi-disciplinary open access archive for the deposit and dissemination of scientific research documents, whether they are published or not. The documents may come from teaching and research institutions in France or abroad, or from public or private research centers.
L'archive ouverte pluridisciplinaire HAL, est destinée au dépôt et à la diffusion de documents scientifiques de niveau recherche, publiés ou non, émanant des établissements d'enseignement et de recherche français ou étrangers, des laboratoires publics ou privés. 
Classification

Physics Abstracts

$72.80-67.90-71.55-61.70 \mathrm{~T}$

\title{
DX centers in AlAs and GaAs-AlAs selectively doped superlattices
}

\author{
S. Ababou, J. J. Marchand, L. Mayet, G. Guillot and F. Mollot (*) \\ Laboratoire de Physique de la Matière (UA CNRS 358), INSA de Lyon, 20 Avenue Albert \\ Einstein, 69621 Villeurbanne Cedex, France \\ (*) Laboratoire de Microstructures et de Microélectronique, C.N.R.S., 196 Avenue H. Ravera, \\ 92220 Bagneux, France
}

(Received 30 April 1990, Revised 22 October and 7 February 1991, accepted 19 March 1991)

\begin{abstract}
Résumé. - Des mesures de spectroscopie capacitives effectuées sur AlAs et sur des superréseaux (SR) GaAs-AlAs dopés sélectivement et épitaxiés par jets moléculaires montrent que le centre DX a une énergie apparente $E_{\mathrm{a}}=0.42 \mathrm{eV}$ aussi bien dans AlAs que dans les SR. Pour la première fois, une étude de la capture menée sur un SR nous permet de remonter à l'énergie d'activation de la section efficace de capture qui est dans notre cas de $0.36 \mathrm{eV}$, ce qui positionne le $\mathrm{DX}$ à $E_{\mathrm{t}} \approx 60 \mathrm{meV}$ de la minibande de conduction. La nature et la position de DX sont discutées en tenant compte de l'énergie et de la concentration du DX pour chaque structure, et nous montrons que le DX observé est lié au silicium dans les couches AlAs.

Abstract. - DX centers have been investigated using deep level transient spectroscopy (DLTS) in Si doped AlAs and in selectively doped GaAs-AlAs superlattices (SLs) grown by molecular beam epitaxy. The activation energy for thermal emission is $E_{\mathrm{a}}=0.42 \mathrm{eV}$ in both the SLs and AlAs layers. For the first time a study of the capture in a SL reveals a capture activation energy $E_{\text {cap }}=0.36 \mathrm{eV}$, which locates the $\mathrm{DX}$ at $E_{\mathrm{t}} \approx 60 \mathrm{meV}$ below the conduction miniband. Taking into account the measured energies and trap concentrations, we show that the DX observed in the SLs lies in the AlAs layers.
\end{abstract}

\section{Introduction.}

DX centers related to the dopan species are present in many III-V semiconductors. They affect the electrical properties of the materials, control the free electron concentration which decreases at liquid nitrogen temperature and cause the persistent photoconductivity phenomenon observed at low temperature $[1,2]$.

The DX center has extensively been studied in GaAlAs alloys which are attractive materials for heterojunction devices such as lasers, light emitting diodes, and various types of transistors. Its thermal activation energy, $E_{\mathrm{a}}$, is the same over a large alloy composition [1], $E_{\mathrm{a}}$ being the sum of the binding energy $E_{\mathrm{t}}$ and the activation energy for thermal capture 
$E_{\text {cap. }}$ In the AlAs layers the deep donor is still not well known since few studies have been performed up to now $[3,4]$.

Despite the large DX apparent concentration in GaAlAs, DX center is not observed on the short period ( $<60 \AA$ ) GaAs-GaAlAs superlattices (SLs) [5], but it appears on the large period (60-100 $\AA$ ) GaAs-GaAlAs SLs [6], where the SL miniband is so thin that the wells become uncoupled under the electric field effect in the space charge region. On the other hand, the DX center is observed in GaAs-AlAs SLs [4,7], and a reduction of its concentration is obtained when these structures are selectively doped $[7,8]$.

In order to get more understanding about the DX center, particularly to determine whether the DX absence in some SLs is due to a resonant state in the miniband or to the band structure modification, electrical characterization including capacitance measurements, thermally stimulated capacitance measurements (TSCAP), deep level transient spectroscopy (DLTS) and capture DLTS have been performed on AlAs layers and GaAs-AlAs selectively doped SLs.

\section{Experimental.}

The AlAs layers have been grown by molecular beam epitaxy on (100) heavily doped GaAs substrates and consist of a $0.5 \mu \mathrm{m}$ Si-doped AlAs layer grown on a Si-doped GaAs buffer layer and covered with a thin GaAs cap. Two samples have been studied : the first one (L1) with a $1 \times 10^{17} \mathrm{~cm}^{-3}$ doping level in the AlAs layer, the doping level of the second one (L2) being $5 \times 10^{17} \mathrm{~cm}^{-3}$ The GaAs-AlAs SLs structures either selectively doped in the middle of GaAs wells (D301) or in AlAs barriers (D305), grown on (100) semi-insulating Cr-doped GaAs substrates, are described in figure 1 . The width of the barriers $L_{\mathrm{B}}$ and of the wells $L_{Z}$ has been determined by double X-ray diffractometry. The free electron concentrations at $300 \mathrm{~K}$ have been deduced from Hall effect measurements. All these characteristics are summarized in table $\mathrm{I}$.

For electrical characterizations, ohmic contacts were made by alloying indium on the backside of the GaAs conducting substrates in the case of AlAs layers and by evaporating Au$\mathrm{Ge}-\mathrm{Ni}$ on the top surface of the sample using a photolithographic process, in the case of semiinsulating $\mathrm{Cr}$-doped substrates. Schottky contacts are obtained by gold evaporation on the front face through a $0.5 \mathrm{~mm}$ diameter dot mask.

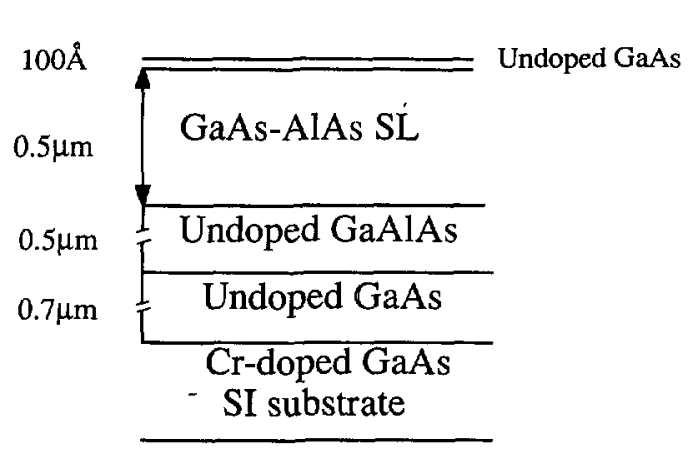

a
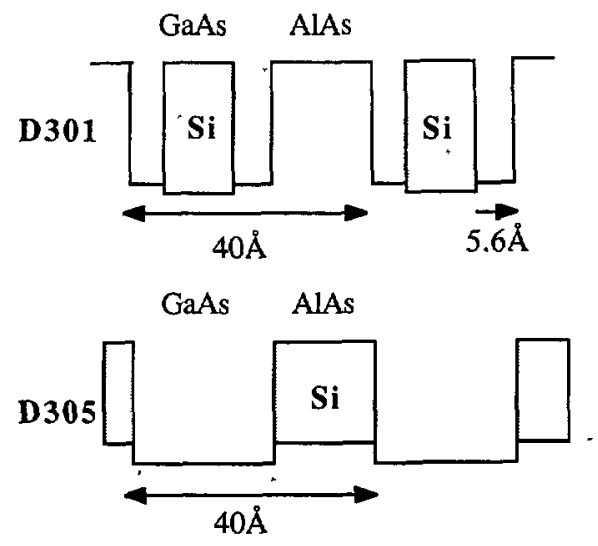

b

Fig. 1. - Schematic diagrams of (a) GaAs-AlAs SL structure and (b) position of the silicon in D301 and D305. 
Table I. - Well and barrier widths $\left(L_{\mathrm{Z}}, L_{\mathrm{B}}\right)$ and free carrier concentration $n_{0}$ deduced from Hall effect measurements at $300 \mathrm{~K}$ for D301 and D305 SLs.

\begin{tabular}{|l|c|c|c|}
\hline & $L_{\mathrm{Z}}(\AA)$ & $L_{\mathrm{B}}(\AA)$ & $n_{0}\left(\mathrm{~cm}^{-3}\right)$ \\
\hline $\mathrm{D} 301$ & 24.2 & 16.8 & $8.6 \times 10^{17}$ \\
\hline $\mathrm{D} 305$ & 22.6 & 16.4 & $8.4 \times 17^{17}$ \\
\hline
\end{tabular}

Capacitance measurements have been performed using a home made capacitance bridge [9] operating at $100 \mathrm{kHz}$ to avoid any effect of the quality factor $Q=R_{\mathrm{s}} C \omega[10,11]$ due to the relatively high serie resistance $R_{\mathrm{s}}$ and capacitance sample $C$, $\omega$ being the pulsation of the measurement signal.

\section{Results and discussion.}

\section{Characterization of AlAs layers.}

1.1 DLTS results. - Deep level transient spectroscopy measurements reveal that no trap is detected on $\mathrm{L} 1$ for temperature ranging from $80 \mathrm{~K}$ to $320 \mathrm{~K}$ and emission rates varying between $0.1 \mathrm{~s}^{-1}$ and $1000 \mathrm{~s}^{-1}$, the lower detection limit of the apparatus being $5 \times 10^{12} \mathrm{~cm}^{-3}$. In L2 sample, DX is detected with a concentration $n_{\mathrm{DX}} \approx 1 \times 10^{15} \mathrm{~cm}^{-3}$ (Fig. 2); from an Arrhénius plot of $T^{2} / e_{\mathrm{n}} v s .1 / T$ one can deduce an activation energy $E_{\mathrm{a}}=(0.42 \pm 0.01) \mathrm{eV}$ for thermal emission and a capture cross section $\sigma_{\infty} \approx 10^{-13} \mathrm{~cm}^{2}$ (Fig. 3 insert).

1.2 Discussion. - A discrepancy exists in the literature concerning the thermal activation energy value of $\mathrm{DX}$ in the AlAs layers [3, 4]. Our determination of $E_{\mathrm{a}}=0.42 \mathrm{eV}$ is coherent with the fact that, for $\mathrm{Ga}_{1-x} \mathrm{Al}_{x} \mathrm{As}$ with $0.04<x<0.95[12-14], E_{\mathrm{a}} \approx 0.42 \mathrm{eV}$.

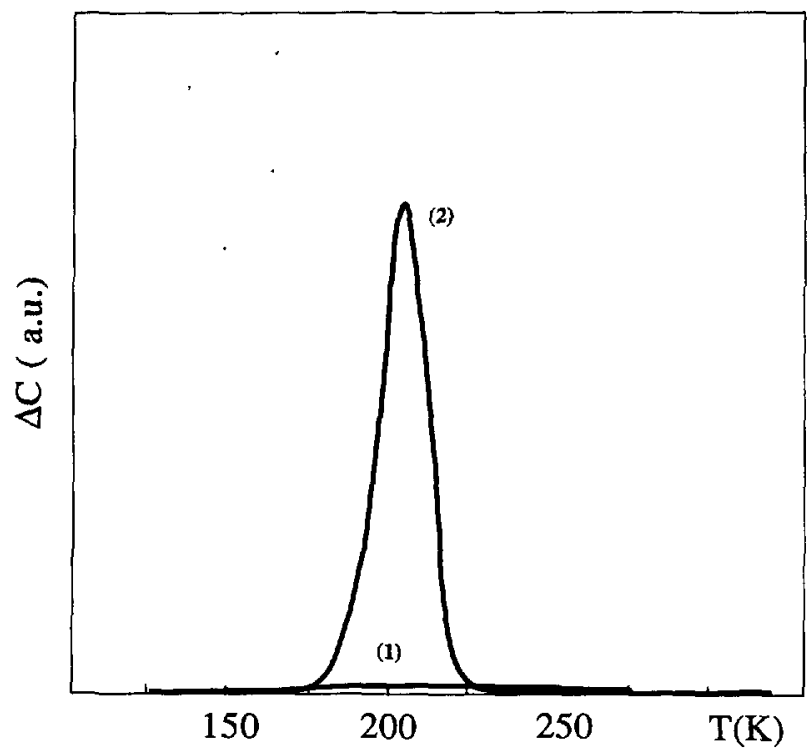

Fig. 2. - Characteristic DLTS spectra obtained (1) on L1, (2) on L2 AlAs layers $e_{n}=100 \mathrm{~s}^{-1}$, $t_{\mathrm{p}}=5 \mathrm{~ms}, V_{\mathrm{r}}=-2 \mathrm{~V}, V_{\mathrm{p}}=0 \mathrm{~V}$. 


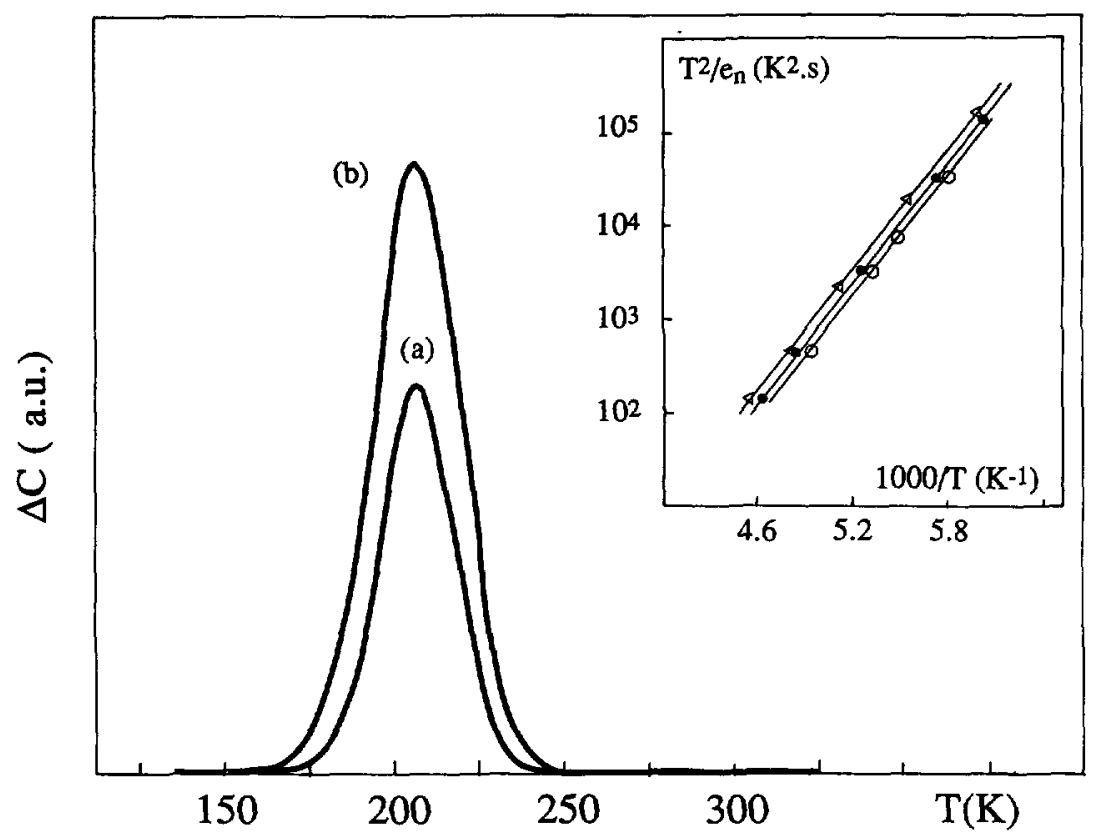

Fig. 3. - Characteristic DLTS spectra on D301 (a) and D305 (b) GaAs-A1As SLs $e_{n}=100 \mathrm{~s}^{-1}$,

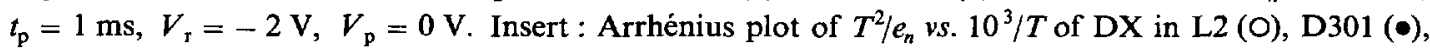
D305 $(\triangle)$.

As the DX center is directly related to the donor in GaAlAs alloys [15], this means that the net ionized donor concentration deduced from $C-V$ measurement is $N_{\mathrm{d}}=N_{\mathrm{DX}} \approx$ [Si], where $N_{\mathrm{DX}}$ is the total DX concentration, the ionization factor being equal to 1 in the space charge region at room temperature, and the total donor concentration is equal to the silicon concentration [Si] in the case of low compensation. On the less doped sample (L1), the number of trapped electrons on the DX center $\left(n_{\mathrm{DX}}\right)$ is below the detection limit, and for a higher doping rate on L2, $n_{\mathrm{DX}}$ starts to be measurable. These values of $n_{\mathrm{DX}}$ indicate a low occupancy factor $n_{\mathrm{DX}} / N_{\mathrm{DX}}$ which is representative of a DX lying near or in resonance with the conduction band [16]. A higher doping pushes the Fermi level towards the $\mathrm{X}$ conduction band and towards the DX level, increasing by this way the occupancy of the trap.

This is consistent with a recent work [3] on AlAs and the negligible persistent photoconductivity already shown on this material [17].

2. Characterization of GaAs-AlAs SLs.

\subsection{Results.}

2.1.1 Emission DLTS. $-C-V$ at $300 \mathrm{~K}$ provides a net donor concentration in agreement with the free electron concentration deduced from Hall effect measurements. From thermally stimulated capacitance measurements (TSCAP) [18], we notice that the whole SL layer is depleted at low temperature on D305 sample. The decrease of the capacitance is less pronounced on D301. The DX center is observed in DLTS spectra for both the SLs (Fig. 3). Its thermal activation energy $\left(E_{\mathrm{a}}\right)$ and capture cross section $\sigma_{\infty}$ are deduced from an Arrhénius plot of $T^{2} / e_{\mathrm{n}} v s .10^{3} / T$ (Fig. 3 insert). The trap concentration is deduced from DLTS measurements at saturating peak amplitude for D301, and from TSCAP for D305 since 
Table II. - Thermal activation energy $E_{\mathrm{a}}$, capture cross section $\sigma_{\infty}$ and trap level concentration $n_{\mathrm{Dx}}$ of the $\mathrm{DX}$ in $\mathrm{D} 301$ and $\mathrm{D} 305$ samples.

\begin{tabular}{|l|c|c|r|}
\hline & $E_{\mathrm{a}}(\mathrm{eV})$ & $\sigma_{\infty}\left(\mathrm{cm}^{2}\right)$ & $n_{\mathrm{DX}}\left(\mathrm{cm}^{-3}\right)$ \\
\hline $\mathrm{D} 301$ & $0.42 \pm 0.01$ & $1.8 \times 10^{-13}$ & $5.1 \times 10^{17}$ \\
\hline $\mathrm{D} 305$ & $0.43 \pm 0.01$ & $1.5 \times 10^{-13}$ & $\approx 8.4 \times 10^{17}$ \\
\hline
\end{tabular}

the DLTS cannot provide this value for high trap concentrations. The DX characteristics $\left(E_{\mathrm{a}}, \sigma_{\infty}, n_{\mathrm{DX}}\right)$ are summarized in table II.

2.1.2 Capture DLTS. - One of the key features of the DX center is its large activation energy for thermal capture. Usually the capture cross section is measured from the filling pulse dependence of the DLTS peak for a fixed temperature. For DX centers however, the capture cross section is so small that the peak cannot be saturated with the filling time normally available. Another method consists in studying the transient during a filling bias voltage. In this case it is necessary to solve the detailed balance equation which gives the time evolution of the electron concentration during the capture [18]. We show in the appendix, that under some well choosen experimental conditions, the transient can be approximated by an exponential.

The capture measurements have been performed on both the superlattices. A home made low frequency pulse generator allowing the application of long filling bias and reverse voltages is used : the time duration of the reverse bias is choosen so that all the traps are ionized before the application of the filling voltage. The capacitance transient is sampled using a classical DLTS double Boxcar technique, the sampling time being higher than $2 \mathrm{~ms}$ and the temperature ranging between $160 \mathrm{~K}$ and $220 \mathrm{~K}$ so that the transient has an exponential form. A typical DLTS spectrum obtained for the capture is illustrated in figure 4 . We can see that the peak keeps the same amplitude for the various rate windows, indicating an exponential form for the transient. An Arrhénius plot of $T^{2} / \tau^{-1} v s .10^{3} / T$ (insert Fig. 4) provides the barrier $E_{\mathrm{b}}$ (see the appendix) which is respectively $(0.36 \pm 0.01) \mathrm{eV}$ and $(0.37 \pm 0.01) \mathrm{eV}$ for D301 and D305. As expected, the capture cross sections $\sigma_{\infty}$ are respectively equal to those deduced from emission.

The energy deduced from this plot is $E_{\mathrm{b}}=E_{\text {cap }}-E_{\mathrm{f}}$, these energies being referred to the bottom of the conduction miniband of the SL, as the lowest conduction state is the minimum of this miniband, even for the electrons moving parallelly to the surface [19] (the peculiar contact geometry of these structures inducing currents perpendicular and parallel to the surface).

The doping level is such that $E_{\mathrm{f}} \approx 0$ at the measurement temperatures, and then $E_{\text {cap }} \approx 0.36 \mathrm{eV}$. This allows us to locate the DX center in these SLs at nearly $60 \mathrm{meV}$ below the bottom of the conduction miniband.

2.2 Discussion. - The DX in GaAs-AlAs SLs has the same activation energy $E_{\mathrm{a}}$ than in GaAlAs alloys for both SLs under study, despite the different dopant position - in the wells or in the barriers - and despite the band structure modification due to the formation of the conduction miniband, which usually changes the emission energy of a defect when placed in a superlattice [20]. The contact geometry does not affect the results since we find the same energy for DX centers in the GaAs-AlAs superlattices where the ohmic contacts are on the backside [21]. 


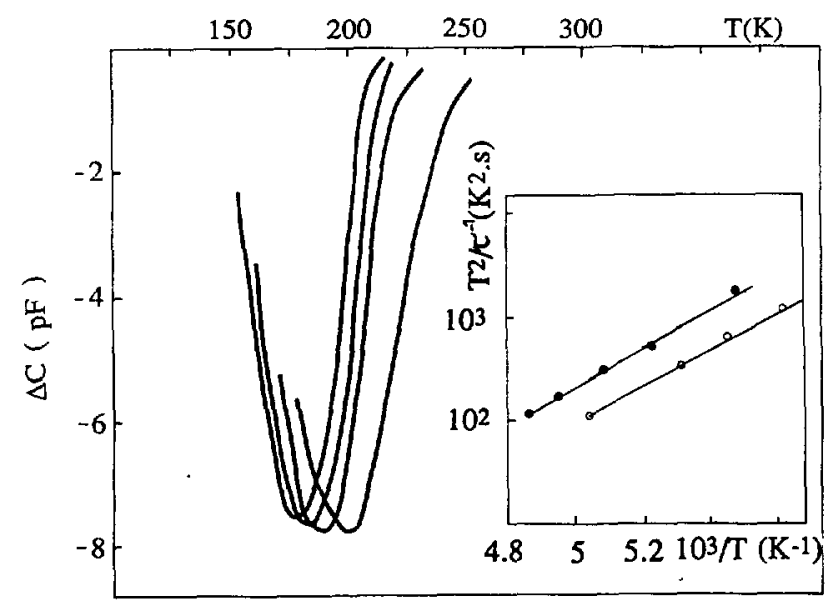

Fig. 4. - Capture DLTS spectra on D301 sample for various rate windows $(25,50,100$ and $\left.345 \mathrm{~s}^{-1}\right) V_{\mathrm{r}}=-2 \mathrm{~V}, V_{\mathrm{p}}=0 \mathrm{~V}$. In insert : Arrhénius plot of $T^{2} / \tau^{-1} v s .10^{3} / T$ for $\mathrm{D} 301(\mathrm{O})$ and D305 (•).

What we notice is the fact that the DX is located at the same energy - 60 meV below the miniband conduction - in both the superlattices. The problem is to know whether the DX lies in the GaAs layers or in the AlAs layers.

2.2.1 The barriers-doped SL. - The DX center in D305 lies below the miniband conduction at a level which corresponds to the DX in the AlAs layers. Indeed, we have shown above that, in bulk AlAs, the DX is resonant or near the X conduction band. This last lies at $0.23 \mathrm{eV}$ from the GaAs $\Gamma$ band (Fig. 5) in the GaAs-AlAs SL. It is then practically $60 \mathrm{meV}$ below the miniband - a theoretical calculation locates this miniband at $0.29-0.30 \mathrm{eV}$ above the GaAs $\Gamma$ band [22] - and it corresponds to the position of the DX we have deduced from the capture study.

2.2.2 The wells-doped SL. - At the opposite of the D305 sample where all the donors act as DX centers, in D301 sample, the trapped electron concentration is lower than the free carrier one $\left(n_{0}\right)$ which, as discussed previously for the case of AlAs, could be due to a resonance of the DX with the conduction miniband. However, the determination of the activation energy $E_{\text {cap }}$ of the capture cross section allowed us to locate this state at $E_{\mathrm{t}} \approx 60 \mathrm{meV}$ below the bottom of the miniband in this SL (Fig. 5), at the same position of the DX in D305. This leads us to think that the DX has the same origin in both the SLs and then is due to the silicon in the AlAs layers. For D301 sample, where only the GaAs wells are intentionnaly doped, the

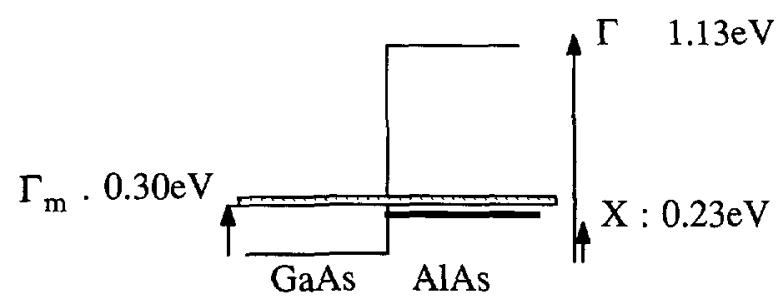

Fig. 5. - Schematic diagram of the conduction band of GaAs-AlAs SL and theoretical position of the $\Gamma_{\mathrm{m}}$ miniband for a conduction band offset $\Delta E_{\mathrm{c} \Gamma}=0.70 \Delta E_{\mathrm{g} \Gamma}$. 
silicon in the AlAs layers can result from a diffusion towards the barriers during the growth which was processed at $580^{\circ} \mathrm{C}$. The fact that only the DX in the AlAs barriers is detected explains the low concentration measured on D301. This is consistent with the reduced apparent DX concentration on wells-doped SLs when the growth temperature is lowered $[7,8]$, and with the increase of the trap concentration after a thermal treatment [23]. In these SLs, the GaAs related $\mathrm{DX}$ is not observed as it lies at $E_{\mathrm{t}} \approx 0.30-0.33 \mathrm{eV}$ above the $\Gamma$ band $[16,24]$ which is a state resonant with the conduction miniband. The hypothesis of a resonant state could also explain why only one peak is observed on these SLs at the opposite of the GaAlAs alloys where the DX appears as a double peak on DLTS measurements [25]. The multi-peak structure in GaAlAs alloys seems to be related to the different atomic configurations around the silicon donor [26]. It has been reported that in the GaAs-AlAs the number of possible configurations of $\mathrm{Al}$ and $\mathrm{Ga}$ around a $\mathrm{Si}$ atom is much more reduced [7]. This would explain the single peak observed in our structures - whatever are the experimental conditions - and the sharp DLTS spectra previously reported by other groups $[7,4]$.

The absence of the DX centers in GaAs-GaAlAs SLs is then due to the state being in resonance with the miniband as in these structures the miniband is a lower energy than in the GaAs-AlAs SLs. Moreover, in uniformly doped GaAs-AlAs SLs, DX appears with a concentration which is as more important as the miniband is at higher energies, allowing then to observe more DX centers [4].

\section{Conclusion.}

DX centers have been characterized in Si-doped AlAs layers and GaAs-AlAs selectively doped superlattices. The activation energy of thermal emission is found to be the same as in GaAlAs alloys, and equals to $0.42 \mathrm{eV}$ in AlAs layer as well as in the SLs. In AlAs, the DX appears to be near or in resonance with the $\mathrm{X}$ conduction band. A detailed analysis of the capture performed on both the SLs shows that the DX center lies below the miniband conduction and that it is due to the silicon in the AlAs layers.

\section{Acknowledgments.}

We wish to thank A. Chomette from CNET Lannion for providing the miniband calculations, and D. Berthier from LPM for his technical assistance.

\section{Appendix A.}

In the case where the DX center controls the free carrier concentration $n$, we have $n_{0}=I N_{\mathrm{Dx}}$ at thermal equilibrium in the neutral region of a Schottky diode, where $I$ is the fraction of ionized deep centers. The evolution with time of the electron concentration during the capture is given by [18] :

$$
n(t, I)=N_{\mathrm{Dx}}\left[\frac{I(1-I)(2-I)}{\exp [(2-I) / I] e_{n} t-(1-I)^{2}}+I\right]
$$

where the emission rate $e_{n}$ is related to the capture rate $c_{n}$ through the equation :

$$
e_{n}(1-I)=c_{n} n_{0} I
$$


As $n_{0}$ is expressed by : $n_{0}=N_{c} \exp \left[-E_{\mathrm{f}} / k T\right]$, for a thermally activated process, which is the case for DX centers, we can write :

$$
c_{n} n_{0}=\langle v\rangle \sigma_{\infty} \exp \left(\frac{-E_{\text {cap }}}{k T}\right) N_{\mathrm{c}} \exp \frac{E_{\mathrm{f}}}{k T}=A \sigma_{\infty} T^{2} \exp \left(\frac{-E_{\mathrm{b}}}{k T}\right)
$$

where $A$ is a constant, the capture activation energy $E_{\text {cap }}$ and Fermi level $E_{\mathrm{f}}$ are referred to the bottom of the conduction band.

For low ionization factors, short times $\left(t<10^{-4} \mathrm{~s}\right)$ and low temperatures (corresponding to low emission rates), the variation of the electron concentration is highly non exponential [27, 28]. However, if $\left(e_{n} t(2-I) / I\right)>1$ then equation (1) reduces to :

$$
n(t, I)=N_{\mathrm{d}}[I(1-I)(2-I) \exp (-t / \tau)+I]
$$

with a time constant of the transient being: $\tau^{-1}=[(2-I) / I] e_{n}=[(2-I) /(1-I)] c_{n} n_{0}$.

For an ionization factor lower than $10^{-1}$, we have to choose experimentally the temperature to have emission rates of the order of $50 \mathrm{~s}^{-1}$, and sampling times greater than $2 \mathrm{~ms}$ so that we can use equation (4) for the capture transient.

\section{References}

[1] Mooney P. M., J. Appl. Phys. 67 (1990) R1.

[2] Lang D. V., in Deep Centers in Semiconductors, edited by S. T. Pantelides (Gordon and Breach Science Publishers, New York) 1986, chapt. 7.

[3] Kasu M., Fujita S. and Sasaki A., J. Appl. Phys. 66 (1989) 3042.

[4] Feng S. L., Zazoui M., Bourgoin J. C. and Mollot F., J. Appl. Phys. 67 (1990) 276.

[5] Bourgoin J. C., Feng S. L., Stievenard D., Letartre X., Barbier E. and Hirtz J. P., Appl. Phys. Lett. 54 (1989) 1115.

[6] Feng S. L., Bourgoin J. C., Mauger A., Stievenard D., Barbier E., Hirtz J.P. and Chomette A., Phys. Rev. B 39 (1989) 13252.

[7] Iwata N., Matsumoto Y., Baba T. and Ogawa M., Jpn. J. Appl. Phys. 25 (1986) L349.

[8] Baba T., Mizutani T. and Ogawa M., J. Appl. Phys. 59 (1986) 526.

[9] Bremond G., Guillot G., Nouailhat A. and Picoli G., J. Appl. Phys. 59 (1986) 2038.

[10] Broniatowski A., Blosse A., Strivastava P. C. and Bourgoin J. C., J. Appl. Phys. 54 (1983) 2907.

[11] Astrova E. V., Lebedev A. A. and Lebedev A. A., Sov. Phys. Semicond. 19 (1985) 850.

[12] Mooney P. M., Caswell N. S. and Wright S. L., J. Appl. Phys. 62 (1987) 4786.

[13] Mooney P. M., Theis T. N., Wright S. L., Appl. Phys. Lett 53 (1989) 2546.

[14] Calleja E., Gomez A., Muñoz E., Appl. Phys. Lett. 52 (1988) 383.

[15] Mizuta M., Tachikwa M., Kukimoto H. and Minomura S., Jpn. J. Appl. Phys. 24 (1985) L143.

Watanabe M. O., Morizuka K., Mashita M., Ashizawa Y., Zohta Y., Jpn. J. Appl. Phys. 23 (1984) L103.

[16] Theis T. N., Mooney P. M. and Wright S. L., Phys. Rev. Lett. 60 (1988) 361.

[17] Chand N., Henderson T., Klem J., Masselink W. T., Fischer R., Chang Y. C. and Morkoc H., Phys. Rev. B 30 (1984) 4481.

[18] IzPura I. and Muñoz E., Appl. Phys. Lett. 55 (1989) 1732.

[19] BASTARD G., in Wave mechanics applied to semiconductor heterostructures, les Editions de Physique, 1988, p. 25.

[20] Bourgoin J. C., Mauger A., Stievenard D., Deveaud B. and Regreny A., Solid State Commun. 62 (1987) 757. 
[21] Ababou S., Benyattou T., Marchand J. J., Mayet L., Guillot G., Mollot F. and Planel R. SPIE's Int. Conf. on the Physical Concepts of Materials for Novel Optoelectronic Devices Application, Nov. 1990, Aachen, RFA, Unpublished.

[22] A Kane-Bastard model is applied for the calculation of the miniband conduction.

[23] Iwata N., Matsumoto Y. and Baba T., Jpn. J. Appl. Phys. 24 (1985) L17.

[24] Baba T., Mizuta M., Fujisawa T., Yoshino J. and Kukimoto H., Jpn. J. Appl. Phys. 28 (1989) L891.

[25] Calleja E., Gomez A., Muñoz E. and Camara P., in Appl. Phys. Lett. 28 (1988) 1877 ;

Calleja E., Gomez A., Criado J. and Muñoz E., Mat. Sci. Forum, 38-41 (1989) 1115.

[26] Mooney P. M., Theis T. N. and Wright S. L., Appl. Phys. Lett. 53 (1989) 2546.

[27] Mooney P. M., Caswell N. S. and Wright S. L., J. Appl. Phys. 62 (1987) 4786.

[28] Bourgoin J. C., Feng S. L. and von Bardeleben H. J., Appl. Phys. Lett. 53 (1988) 1841. 